\title{
A Colorization Algorithm with Artifacts Suppression on Real Time Video
}

\author{
Sneha P. Mete ${ }^{1}$, Mayur S. Dhait ${ }^{2}$ \\ ${ }^{1}$ M. Tech. Student Computer Science and Engineering, Agnihotri College of Engineering Nagthana Road, Sindhi (Meghe), Wardha, \\ RTMNU University, Maharashtra, India \\ ${ }^{2}$ Assistant Professor, Department of Electronics and Communication Engineering, Agnihotri College of Engineering Nagthana Road, \\ Sindhi (Meghe), Wardha, RTMNU University, Maharashtra, India
}

\begin{abstract}
Color transfers between images are difficult operation in images editing but easily suffers from some corruptive artifacts in the mapping process. In this paper, a color transfer approach with corruptive artifacts suppression is represent, which performs iterative probabilistic color mapping and this Paper extend the technique to colorize the videos. It is done with the help of self-learning filtering scheme and multiscale detail manipulation scheme, which minimizes Kullback-Leibler distance. This will help us to colorize both already colored and gray scale videos. This method achieves a sound grain suppression which means it prevent from being published then a color fidelity that means the degree of exactness with which something is copied or reproduced and detail appearance. First, an iterative probabilistic color mapping is applied to construct the mapping relationship between the reference and target frames. Then, a self learning filtering scheme is applied into the transfer process to prevent from artifacts and extract details. The transferred output and the extracted multi-levels details are integrated. This is done by the measurement minimization to yield the final result.
\end{abstract}

Keywords: Colored/ gray scale videos, Corruptive Artifacts, Grain, Color Fidelity

\section{Introduction}

Now a Day using of images and videos are very common. In any web page, uses of videos/images are used to getting the things very clearly. Color manipulation is one of the most common tasks in image editing. While artists resort to photo editing tools to manually adjust color appearance, automatic color appearance adjustment is still of high demand, owing to the inherent difficulties to handle complex structures ubiquitous in natural images.. Representative approaches include classical histogram matching, statistical transfer [3], $\mathrm{N}$-dimensional probability density function transfer [4], gradient-preserving transfer [5], non-rigid dense correspondence transfer [6], progressive transfer [7], to list a few. Example-based color transfer is a critical operation in image editing but easily suffers from some corruptive artifacts in the mapping process.example-based color transfer [2], which aims at copying the color appearance from a given "example" to a target grayscale or color image, is the most effective way to tackle the problem. Rapid development has been witnessed in the last decade in the field of color transfer consider Fig. 1 shows remarkable artifacts as follows

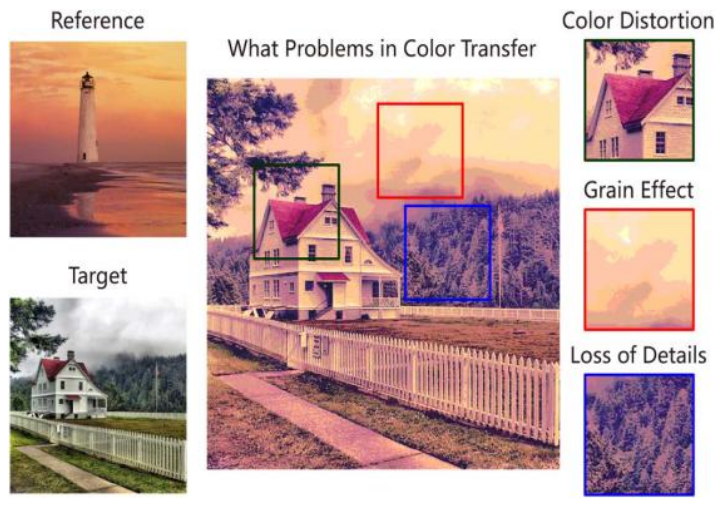

Fig. 1. Example-based color transfer [1] is an image manipulation technique, but it would produce some unexpected artifacts due the intensity of images.

Color distortion. Some unexpected colors appear which are not present in the reference image.

Grain effect. A phenomenon appears due to enhancing the noise level of the picture under the stretched mapping. Commonly, it looks like some noises or irregular blocks.

Loss of details. The fine-level details in the target image are missed after the color transfer.

This paper proposed a special color transfer framework with corruptive artifacts suppression, which performs iterative probabilistic color mapping with self-learning filtering scheme and multiscale detail manipulation scheme in minimizing the normalized Kullback-Leibler distance. First, an iterative probabilistic color mapping is applied to construct the mapping relationship between the reference and target images. Then, a self-learning filtering scheme is applied into the transfer process to prevent from artifacts and 


\section{International Journal of Science and Research (IJSR) \\ ISSN (Online): 2319-7064}

Index Copernicus Value (2013): 6.14 | Impact Factor (2015): 6.391

extract details. The transferred output and the extracted multi-levels details are integrated by the measurement minimization to yield the final result. Our framework achieves a good sound grain suppression, color fidelity and detail appearance smoothly and sharply.

\section{Literature Survey}

1. In this paper, The author proposed a novel unified color transfer framework with corruptive artifacts suppression, which performs iterative probabilistic color mapping with self-learning filtering scheme and multiscale detail manipulation scheme in minimizing the normalized Kullback-Leibler distance. First, an iterative probabilistic color mapping is applied to construct the mapping relationship between the reference and target images. Then, aself-learning filtering scheme is applied into the transfer process to prevent from artifacts and extract details. The transferred output and the extracted multi-levels details are integrated by the measurement minimization to yield the final result. Our framework achieves a sound grain suppression, color fidelity and detail appearance seamlessly [1]

2. In this paper, the author proposed introduced a general technique for "colorizing" greyscale images by transferring color between a source, color image and a destination, greyscale image. Although the general problem of adding chromatic values to a greyscale image has no exact, objective solution, the current approach attempts to provide a method to help minimize the amount of human labor required for this task. Rather than choosing RGB colors from a palette to color individual components, we transfer the entire color "mood" of the source to the target image by matching luminance and texture information between the images. We choose to transfer only chromatic information and retain the original luminance values of the target image. Further, the procedure is enhanced by allowing the user to match areas of the two images with rectangular swatches. This show that this simple technique can be successfully applied to a variety of images and video, provided that texture and luminance are sufficiently distinct. The images generated demonstrate the potential and utility of our technique in a diverse set of application domains. Isoline Based Image Colorization Colorization is a process of adding colors to black and white images and videos [2].

3. In this paper, the author proposed One of the most common tasks in image processing is to alter an image s color. Often this means removing a dominant and undesirable color cast, such as the yellow in photos taken under incandescent illumination. This article describes a method for a more general form of color correction that borrows one image es color characteristics from another. We can imagine many methods for applying the colors of one image to another. Our goal is to do so with a simple algorithm, and our core strategy is to choose a suitable color pace and then to apply simple operations there. When a typical three channel image is represented in any of the most well-known color spaces, there will be correlations between the different

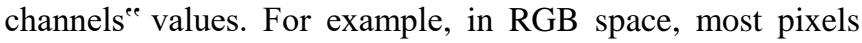
will have large values for the red and green channel if the blue channel is large. This implies that if we want to change the appearance of a pixeles color in a coherent way, we must modify all color channels in tandem. This complicates any color modification process [3].

4. In this paper, the author proposed article proposes an original method to estimate a continuous transformation that maps one $\mathrm{N}$-dimensional distribution to another. The method is iterative, non-linear, and is shown to converge. Only 1D marginal distribution is used in the estimation process, hence involving low computation costs. As an illustration this mapping is applied to color transfer between two images of different contents. The paper also serves as a central focal point for collecting together the research activity in this area and relating it to the important problem of automated color grading [4].

5. In this paper, a novel color transfer algorithm is presented to resolve the fidelity problem of color transfer in terms of scene details and colors. It's well known that human visual system is more sensitive to local intensity differences than to intensity itself. We thus consider that preserving the color gradient is necessary for scene fidelity. We formulate the color transfer problem as an optimization problem and solve it in two steps histogram matching and a gradient-preserving optimization. Following the idea of the fidelity in terms of color and gradient, we also propose a metric for objectively evaluating the performance of example-based color transfer algorithms [5].

\section{Proposed Approach}

An example-based color transfer, which goal at copying the color present from a reference "example" to a target grayscale or color image but it would produce some unexpected artifacts due to the complexity of color mapping. Grain effect, color distortion and loss of details appear in the output. So, this paper introduced to reduce that artifacts by using different technique and this paper extend the framework to video editing. This flow shown in fig.2. Ideally, color transfer between reference frames and target frames should satisfy the following aims.

1)The degree of Exactness with which something is copied or reproduced.

2)Grain suppression means No visual artifacts like grain or blocky artifacts should be generated in the target frames.

3)Detail preservation that is Details in the original target frames should be preserved after the transfer of frames.

This paper present a special framework for example-based color transfer, which aims to achieve simultaneously grain suppression, color fidelity and detail preservation. In propose work, we are working with videos and we will be using example based coloring technique. The artifacts suppression will be minimize by using special color transfer framework. This performs iterative probabilistic color mapping with selflearning filtering scheme and multiscale detail manipulation scheme in minimizing the normalized Kullback-Leibler distance. First, an iterative probabilistic color mapping is applied to construct the mapping relationship between the 


\section{International Journal of Science and Research (IJSR) \\ ISSN (Online): 2319-7064}

Index Copernicus Value (2013): 6.14 | Impact Factor (2015): 6.391

reference and target frames. Then, a self-learning filtering scheme is applied into the transfer process to prevent from artifacts and extract details. The transferred output and the extracted multi-levels details are integrated by the measurement minimization to yield the final result. we are working with Videos. Video is collection of many frames .here we present twenty frames and produced Video. Then color will be transfer from frame to frame. We are working with HSV(Hue Saturation Value).To colorize every frames .we first transfer color from a reference color frame to a single target frame. Then all frames in the video can be colorize using the same colorized target swatches used in the single frame.

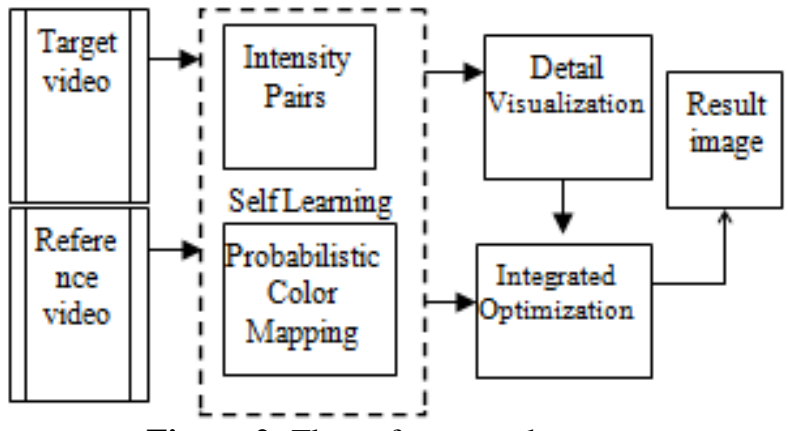

Figure 2: Flow of proposed system

First, a probabilistic mapping is iteratively applied to generate coarse color mapping. After this it reduce the $\mathrm{N}$ dimensional probability distribution of both reference and target frames to a one dimensional probability distribution pair. This method can match the color distribution of the target frames to the reference frames. Second, the selflearning filtering method is used into the procedure of color mapping. The intensity channels are taken as the learning example for filtering. This can be achieving by converting target frames into uncorrelated space, which is further applied to the mapped result.

\section{Methodology}

This Paper proposes a technique to colorized videos .This will help us to colorize both already colored and gray scale videos. This Paper will use example base color transfer technique which aims at copying the color appearance from a given "reference" to a target grayscale or color frames, is the most effective way to tackle the problem. This Paper proposed a novel color transfer framework to deal with these corruptive artifacts by integrated a self-learning filtering scheme into the iterative probabilistic color mapping model. Our framework not only prevents the color distortion and grain effect but also achieves the effect of preserve detail or enhancing and clarity. In addition, to evaluate the quality of color transfer, we proposed a convergence analysis, shape analysis of color distribution, visual comparison and user investigation. By the experimental analyses in the objective and subjective data, we found that our framework had a better performance than the other especially in dealing with the grain effect, color distortion, and loss of details.The overview of our framework is as follows.
Color mapping stage. A probabilistic color mapping is applied to achieve the basic color corresponding and a selflearning filtering is embedded to avoid the artifacts and separate the transferred target into levels.

Detail manipulation stage. A multiscale detail manipulation scheme is applied to preserve or enhance the details.

Integrated optimization stage. The transferred result and the modified details are combined into an optimization solution with the normalization Kullback-Leibler measurement to yield the final output.

\subsection{Kullback-Leibler Distance for Color Transfer}

The Kullback-Leibler distance (K-L) can measure the similarity between two completely determined probability distributions. Here, we apply it to measure the difference between the reference and transferred result in color transfer.

\subsection{Iterative Probabilistic Color Mapping}

First, an iterative probabilistic color mapping is applied to construct the mapping relationship between the reference and target frames.

\subsection{Self-learning Filtering Scheme}

This present a self-learning filtering scheme and incorporate it into the afore mentioned iterative probabilistic color mapping. Firstly, assume the transferred result $g$ and its filtered output $\hat{g}$ are divided into a series of $9 \times 9$ patches, and each patch-pair has 1-to-1 corresponding relationship.

\subsection{Multiscale Detail Manipulation Scheme}

Details in the original target should be preserved after the transfer. Actually, details often correlate to the style appearance, and this characteristic is significant to the colorrelated applications. Since we have incorporated the selflearning filtering scheme into the color mapping, we can exploit its property of edge-preserving decomposition to extract the details while compensating or enhancing them in the transferred output. In our framework, $d^{k}$-levels details are obtained by iteratively applying the self-learning filtering scheme.

\subsection{Integrated optimization Framework}

We presented the K-L distance can be used to evaluate the similarity between the color distribution of the reference frames and that of the transferred frames. A systematic approach for development of a reliable optimization framework to address the optimal design of integrated bio refineries in the face of uncertainty is presented. In the current formulation, a distributed strategy which is composed of different layers including strategic optimization, risk management, detailed mechanistic modeling, and operational level optimization is applied.. 


\section{International Journal of Science and Research (IJSR) \\ ISSN (Online): 2319-7064}

Index Copernicus Value (2013): 6.14 | Impact Factor (2015): 6.391

\section{Implementation}

\subsection{Collection of images for colorization}

This first module includes various images like grayscale images and color images for colorization. Following figure shows the welcome figure from which we can colorize grayscale images. we can select different images from button image colorization. We can also includes images other than this images. This images may be static or real time. But these images are totally static.

\section{Image Colorization Image Colorization \\ About Project \\ Exit}

Figure 5.1.1: Welcome figure

This second figure shows the what actually select gray images from button select gray image and select color images from button select color image for colorization .and also we can get information about selected images

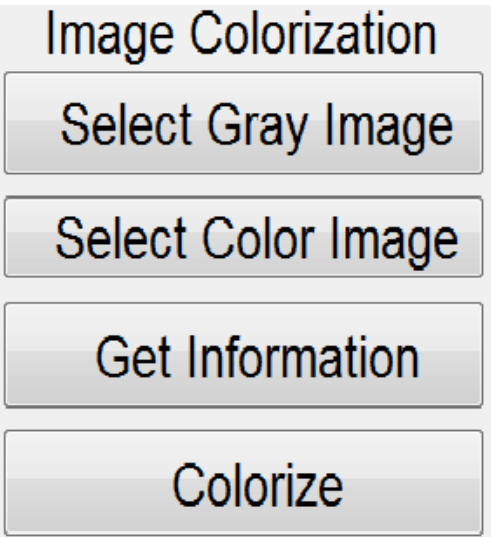

Figure 5.1.2: Image Colorization

Then third figure shows the how to browse the gray images from different folders which we want.

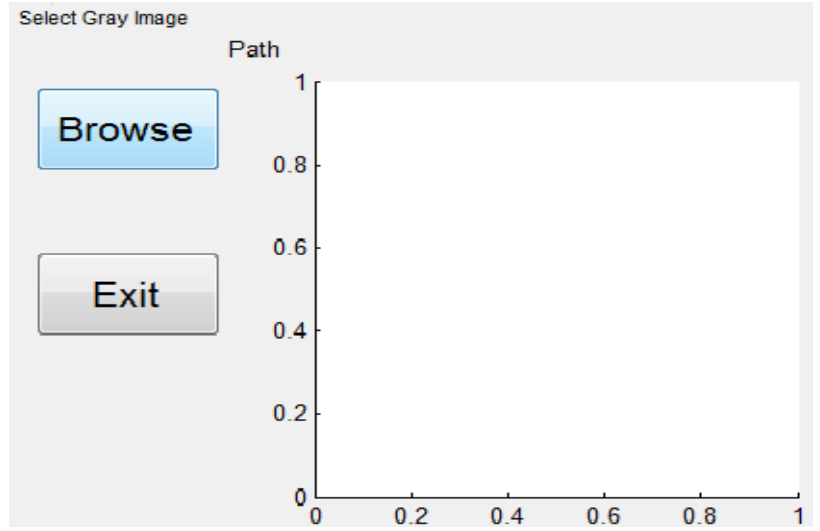

Figure 5.1.3: Browse gray Image
Following fourth and fifth figure represent the selected gray image of flowers and color image of tulips and also represents path from which images selected.

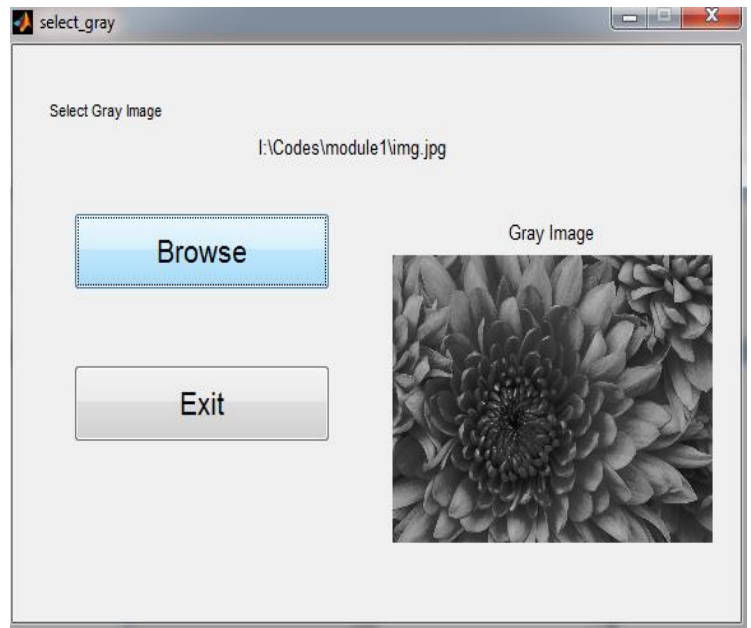

Figure 5.1.4: Selected gray Image

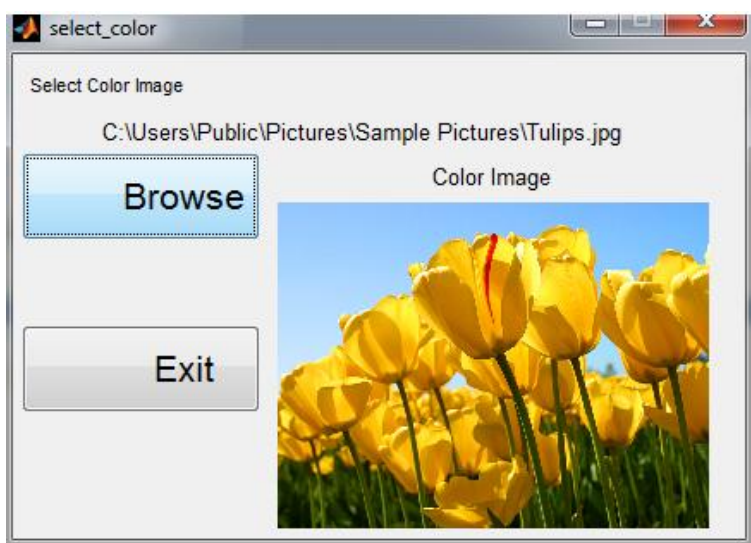

Figure 5.1.5: Select color Image

Last figure shows the information about selected gray images and color images. Details contain chrominance, luminance, type, coding method, size, format, depth.

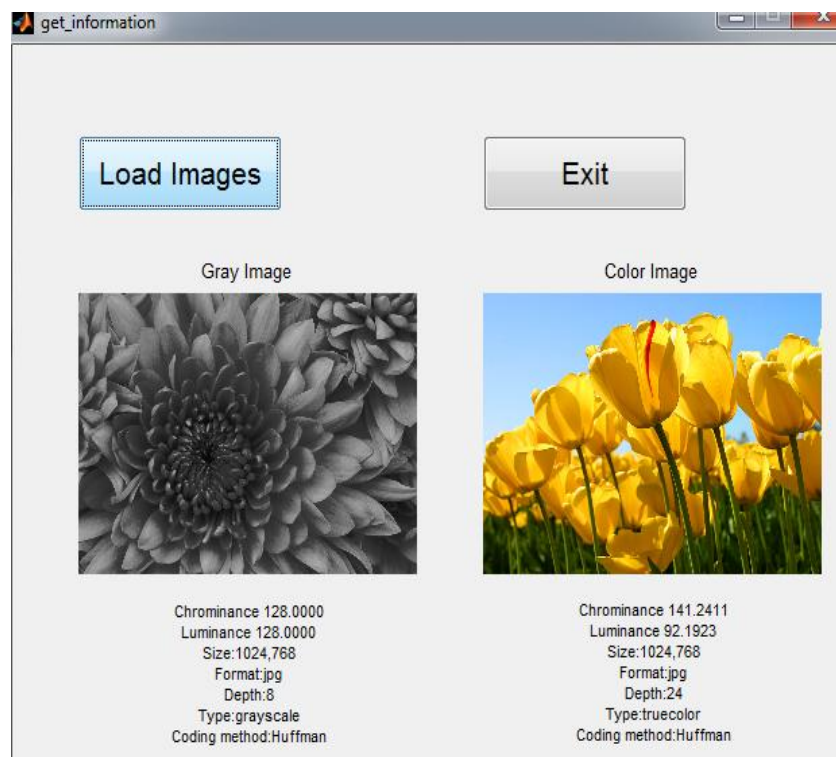

Figure 5.1.6: Selected gray and color images and Get Information about Images 


\section{International Journal of Science and Research (IJSR) \\ ISSN (Online): 2319-7064}

Index Copernicus Value (2013): 6.14 $\mid$ Impact Factor (2015): 6.391

5.2. Texture analysis of the gray scale image and compare with the colored image

This second module represents the matching pixels between two images i.e. color images and gray images. That means maximum match means min difference pixels between this two images. which shows the matching pixels and shows transferred images as a output.

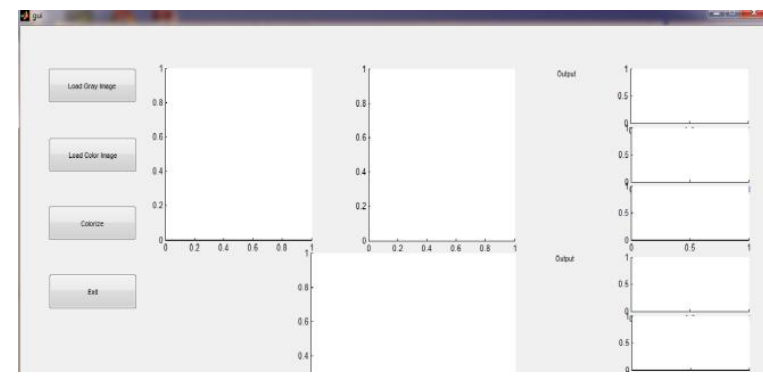

Figure 5.2.1: Select gray and color image

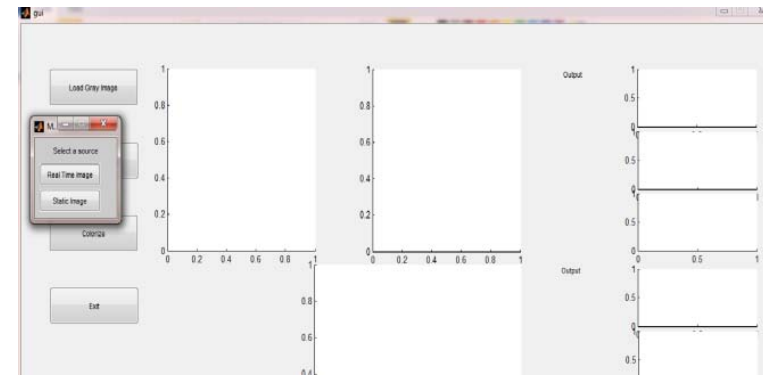

Figure 5.2.2.selecting source image

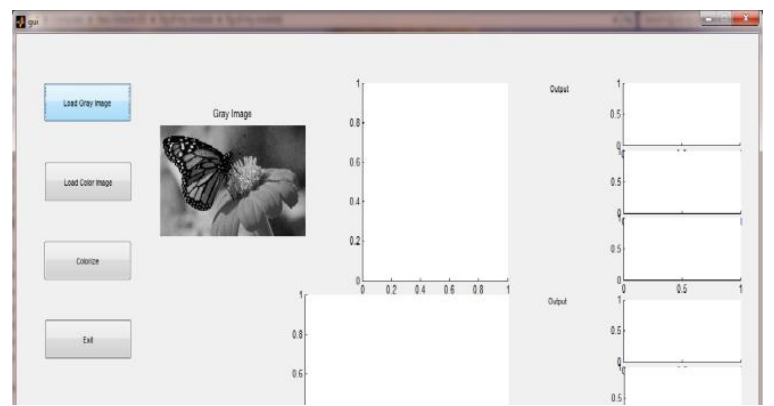

Figure 5.2.3.selected gray image

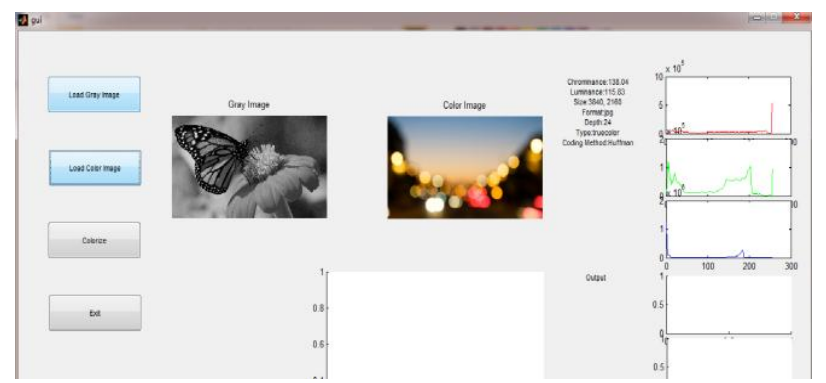

Figure 5.2.4.selected color image

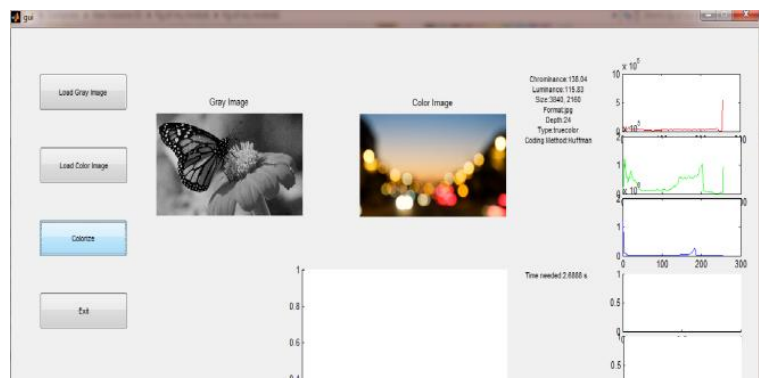

Figure 5.3.5.for match pixels between gray and color images

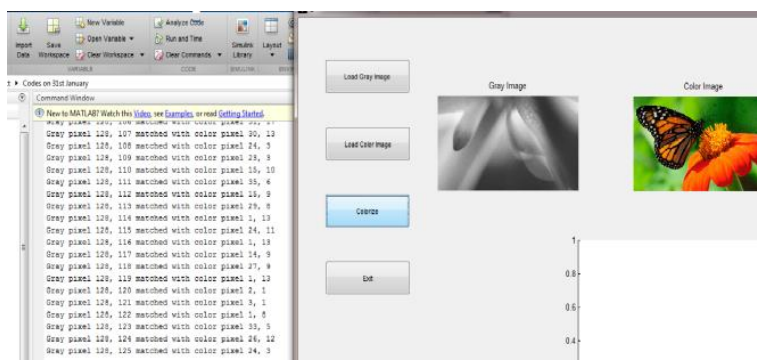

Figure 5.2.6. matched pixels

\section{Conclusion}

This paper concludes that the planned framework minimizes these corruptive artifacts using a color transfer method. Planned method not only prevents the color distortion and grain effect in the process of color transfer, but also preserves the detail and enhances the videos smoothly, clearly. Main thing is that planned method not only transfer color from images to images but also transfer color from video to other video. That means it modify the technique to transfer color in to the videos from gray scale or color videos. Here user can first select reference and target frames one by one from which color will be transfer then using multiple method video will be colorized. This will help us to colorize both already colored and gray scale videos.

\section{References}

[1] Zhuo Su, Kun Zeng, Li Liu, Bo Li, and Xiaonan Luo, "Corruptive Artifacts Suppression for Example-based Color Transfer, " IEEE TRANSACTIONS ON MULTIMEDIA, VOL. 11, NO. 1, JANUARY 2013.

[2] E. Reinhard, M. Ashikhmin, B. Gooch, and P. Shirley, "Color transfer between images, " IEEE Computer Graphics and Applications, vol. 21, no. 5, pp. 3441, 2001.

[3] T. Welsh, M. Ashikhmin, and K. Mueller, "Transferring color to greyscale images, " ACM Trans. Graph., vol. 21, no. 3, pp. 277-280, 2002.

[4] T. Pouli and E. Reinhard, "Example-based color image manipulation and enhancement, " in Proc. ACM SIGGRAPH 2012Courses, ser. SIGGRAPH „12, 2012, pp. 3:1-3:62.

[5] F. Pitié, A. Kokaram, and R. Dahyot, "Automated color grading using color distribution transfer, " Comput. Vision ImageUnderstand., vol. 107, no. 1-2, pp. 123137, 2007.

[6] F. Pitie, A. C. Kokaram, and R. Dahyot, "N-dimensional probablility density function transfer and its application to color transfer, " in Proceedings of the 10th IEEE 
International Conference on Computer Vision, vol. 2, 2005, pp. 14341439.

[7] X. Xiao and L. Ma, "Gradient-preserving color transfer, " Computer Graphics Forum, vol. 28, no. 7, pp. 18791886, 2009.

[8] Y. HaCohen, E. Shechtman, D. B. Goldman, and D. Lischinski, "Nonrigid dense correspondence with applications for image enhancement, " ACM Transactions on Graphics, vol. 30, no. 4, pp. 70:170:10, 2011.

[9] T. Pouli and E. Reinhard, "Progressive color transfer for images of arbitrary dynamic range, " Computers Graphics, vol. 35, no. 1, pp. 6780, 2011.

[10]R. C. Gonzalez and R. E. Woods, Digital Image Processing, 3rd ed. Prentice Hall, Upper Saddle River, NJ., 2008.

[11] Y. Chang, S. Saito, K. Uchikawa, and M. Nakajima, "Example-based color stylization of images, " ACM Trans. Appl. Percept., vol. 2, no. 3, pp. 322-345, 2005.

[12] Y. Tai, J. Jia, and C. Tang, "Soft color segmentation and its applications, " IEEE Trans. Pattern Anal. Mach. Intell., vol. 29, no. 9, pp. 1520-1537, 2007.

[13]A. Abadpour and S. Kasaei, "An efficient PCA-based color transfer method, " J. Visual Commun. Image Represent., vol. 18, no. 1, pp. 15-34, 2007.

[14] F. Pitié, A. Kokaram, and R. Dahyot, "Automated color grading using color distribution transfer, " Comput. Vision Image Understand., vol. 107, no. 1-2, pp. 123137, 2007.

[15] W. Dong, G. Bao, X. Zhang, and J.-C. Paul, "Fast local color transfer via dominant colors mapping, "ACM SIGGRAPH Asia 2010Sketches, pp. 46:1-46:2, 2010. 